\title{
Biodegradation Properties of Poly (Lactic) Acid Reinforced by Kenaf Fibers
}

\author{
S.N. SURIP ${ }^{a *}$, W.N.R. WAN JAAFAR ${ }^{a}$, N.N. AZMI ${ }^{a}$ AND N.A. HASSAN ${ }^{b}$ \\ ${ }^{a}$ Bio-Composites Technology Programme, \\ Faculty of Applied Sciences, Universiti Teknologi MARA, 40450 Shah Alam, Malaysia \\ ${ }^{b}$ Department of Manufacturing and Materials Engineering, Kulliyyah of Engineering, \\ International Islamic University Malaysia, P.O. Box 10, 50728 Kuala Lumpur, Malaysia
}

\begin{abstract}
This study was conducted to study the degradation of poly (lactic acid) and its composites under natural landfill burial. Composites reinforced with natural fibres were expected to degrade faster than polymer itself. PLA was compounded with kenaf bast fibre (KBC) and kenaf core fibre (KCC) with twin screw extrusion at temperature range $150-160^{\circ} \mathrm{C}$ and being compression moulded at $170{ }^{\circ} \mathrm{C}$ for 8 minutes. Samples were then cut prior to testing by burying under composting area in UiTM Shah Alam, Selangor, Malaysia for 6 month period. Samples were measured and observed monthly for the degradation of composites by weight loss and microscopic observation. As expected weight loss for kenaf bast composite (KBC) and kenaf core composite (KCC) was found to be higher, $15.9 \%$ and $17.1 \%$ respectively, than that of pure PLA of only $4.14 \%$. Microscopic observation confirms degradation has occurred on surface of composites by making cracks, holes, and black spots on all samples, however degradation was more obvious on composites. FTIR analysis shows that spectra of exposed composites were reduced compared to those of unexposed composites.
\end{abstract}

DOI: 10.12693/APhysPolA.129.835

PACS/topics: 87.85.jf

\section{Introduction}

Poly lactic acid is one of the degradable polyesters that has been widely used in various applications. PLA is completely mineralized to $\mathrm{CO}_{2}$, water and a small amount of biomass after four to six weeks in composting conditions, at approximately $60^{\circ} \mathrm{C}[1-4]$. Degradation of poly lactic acid was studied to investigate the best method for its degradation that would assist in reducing the environmental pollution. Although it is degradable, the degradation of polyester could take longer time than expected. It is due to the thousands of polymer chain that should be broken before the further process. Zhang et al. [5]; Auras et al. [6]; Tokiva and Jarerat [7] have studied and have proven that degradation of PLA were completed in aerobic (composting) or anaerobic (biomethanation) environments over a period of six month to five years. The degradation of PLA was affected by various factors including moisture, heat, light, microorganism, etc. Other than that, the size of the samples was also an important factor, where smallersized samples were easier to be degraded than larger samples due to higher surface of exposure to microorganism attack $[8,9]$.

\section{Materials and methods \\ 2.1. Materials}

PLA used in this study was supplied by Shenzen Bright China Industrial Co., Ltd., China. PLA was

*corresponding author; e-mail: snorasmah@salam.uitm.edu.my reinforced with 2 weight percent fibre loading. Kenaf fibre was pre-mixed with PLA before placing into the extrusion apparatus (Prism TSE System Type DSR 28 (No. 966195M02)) with five heating zones at temperature range of $150-160^{\circ} \mathrm{C}$. After that, the extrudate was pelletilized using Prism TSE System cutter (No. 2094). Then, the pellets were placed inside the desiccator before the compression moulding. Collected pellets were compression moulded (Hung Ta Instrument Co. Ltd Type HT-8122 A (No. 2260)) at temperature of $175^{\circ} \mathrm{C}$ for eight minutes. Subsequently, composites were left to cool under the applied pressure. Afterwards, composites were cut for landfill burial testing.

\subsection{Landfill burial testing}

Landfill testing was conducted by adopting ASTM G160-12 with a few modifications done following the method of Rudnik and Briassoulis [10] and Fukushima et al. [11]. Modifications were adopted because the landfill burial test was natural, whereas the ASTM G160-12 is a laboratory landfill burial. Samples were buried at a depth of $15 \mathrm{~cm}$ at composting area in
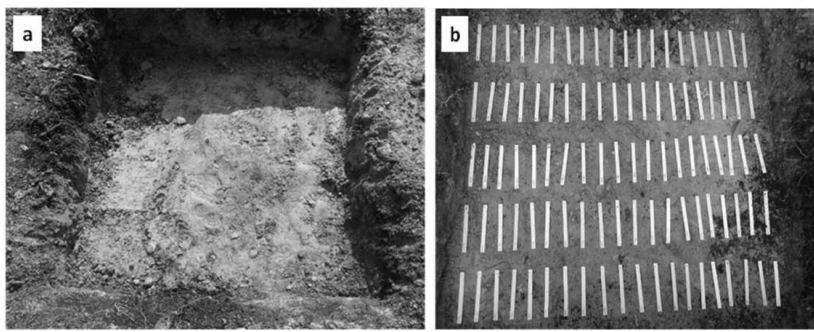

Fig. 1. Landfill burial test set up. (a) Area of testing. (b) The arrangement of samples before burial. 
UiTM, Shah Alam. Irrigation cycle was applied in which one day was with irrigation and one day was without irrigation. However, the irrigation was interrupted during raining period. The test set up is shown in Fig. 1, where Fig. 1a shows the testing area and Fig. 1b shows the arrangement of samples before the burial.

\subsection{Characterization}

To measure the rate of degradability of the composites, weight loss of the samples was measured every month for a six months period. Microscopic observation was done to observe the changes on the surface of the samples after being buried in the soil.

\section{Results and discussion}

Figure 2 shows the soil temperature and $\mathrm{pH}$ for the testing period. Soil $\mathrm{pH}$ has shown an inconsistent trend, where it was ranging from 4 to 7 during the testing period. According to Andersson et al. [12] the decreasing of $\mathrm{pH}$ was due to the formation of acidic degradation products that migrated into the soil. While the increasing of the soil $\mathrm{pH}$ was due to higher soil water content, determined by moisture meter, which has neutralized the acidity of the soil. In addition, the degradation products of PLA were carbon dioxide, water and humus [11, 13]. Soil water content for October 2013 till

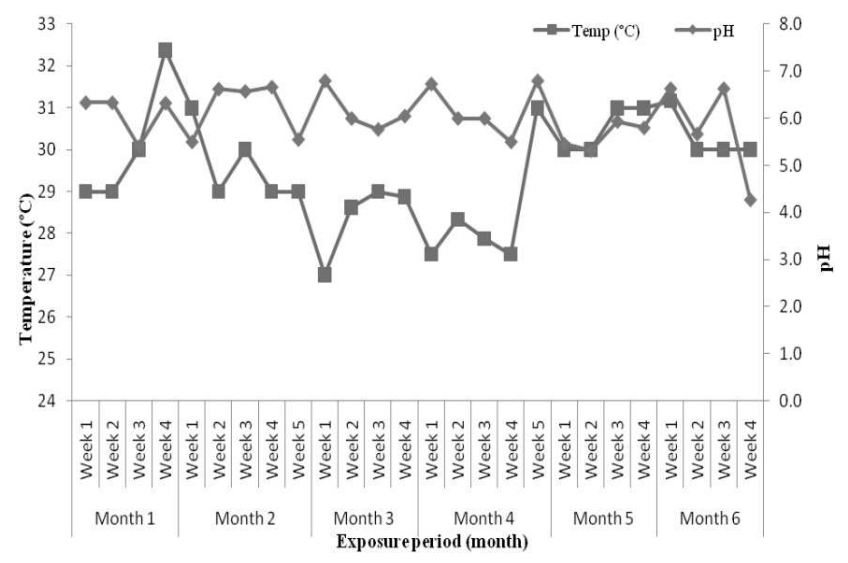

Fig. 2. Soil temperature and $\mathrm{pH}$ of landfill testing.

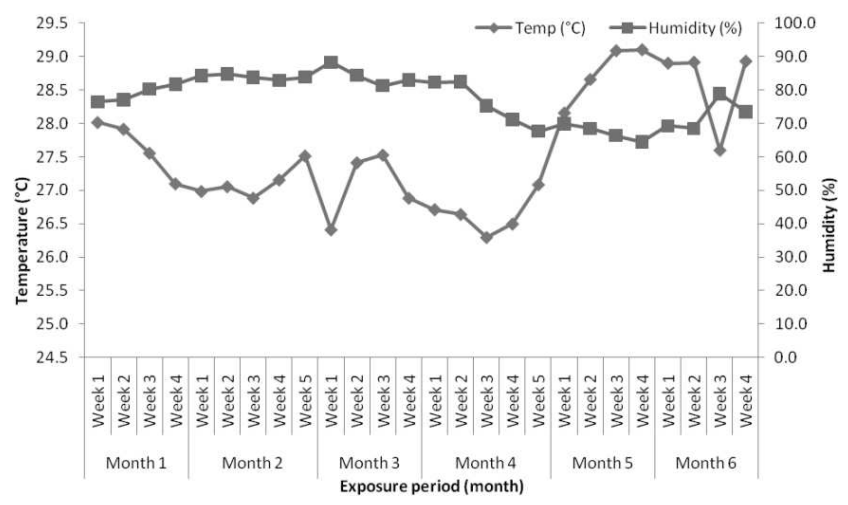

Fig. 3. Air temperature and humidity.
March 2014 was 99\%,98\%,95\%, 90\%, 43\% and $77 \%$ respectively. It shows that the soil in the testing area was moist for the entire period. The meteorological data (air temperature and air humidity) is reported in Fig. 3. Total rainfall was $4.50 \mathrm{~mm}, 13.72 \mathrm{~mm}$, $10.34 \mathrm{~mm}, 4.74 \mathrm{~mm}, 0.25 \mathrm{~mm}$, and $5.06 \mathrm{~mm}$ from October 2013 till March 2014, respectively.

Percentage of weight loss of KBC and KCC are shown in Fig. 4. The weight loss of pure PLA and composites was gradually increasing from the first month exposure until the sixth month. The degradation of the KBC and KCC was similar. Weight loss of pure PLA shows the lowest amount of weight loss among others. It indicates that incorporation of natural fibre assists in the degradation of the composites, since the cellulose fibre attracts the attacking microorganism. Lu et al. [14] explains that plant fibres are degraded into carbohydrate by numerous micro-organisms existing in nature, for example, brown rot fungi would decompose cellulose and hemicelluloses while white rot fungi would decompose lignin.

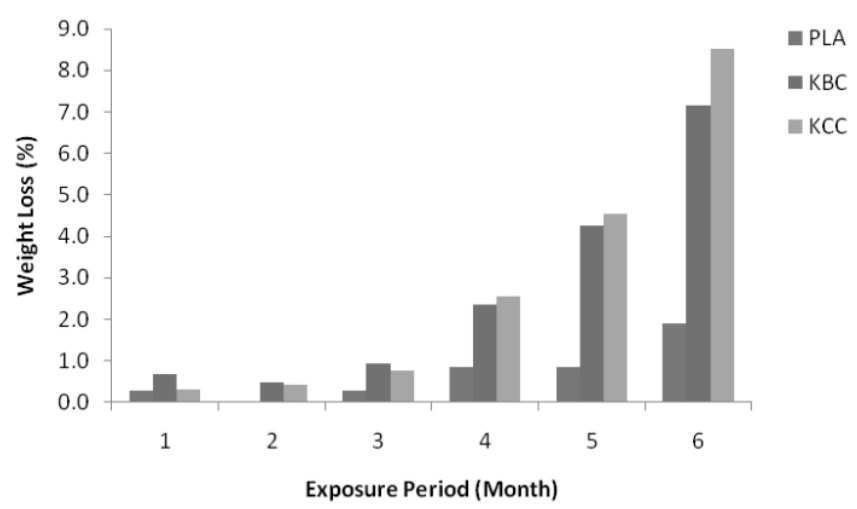

Fig. 4. Weight loss of KBC and KCC at landfill burial test.

Figure 5 shows the comparison between control samples and samples after six months landfill burial. KBC, KCC and pure PLA were initially having smooth surface before testing. Six months of landfill burial shows changes of colour and surface deformation on the entire samples surface for $\mathrm{KBC}, \mathrm{KCC}$ and pure PLA. The whitening effect indicates the beginning of hydrolytic degradation of the polymer chain, that changes the refraction index of the samples due to water absorption and presence of byproducts of hydrolytic process $[15,16]$. Shogren et al. [17] indicated that colour changes were due to the beginning of microbial growth on the samples that lead to degradation of the samples.

With the presence of holes, surface cracks and black spots on the surface of KBC, KCC and pure PLA, the strength of the composites was reduced. Although no mechanical testing was conducted, while handling the samples, the brittleness of the samples was increasing with the longer time of exposure. Samples became soft and broke even under small applied forces. Similar result was reported by Rudnik and Briassoulis [10], they reported 


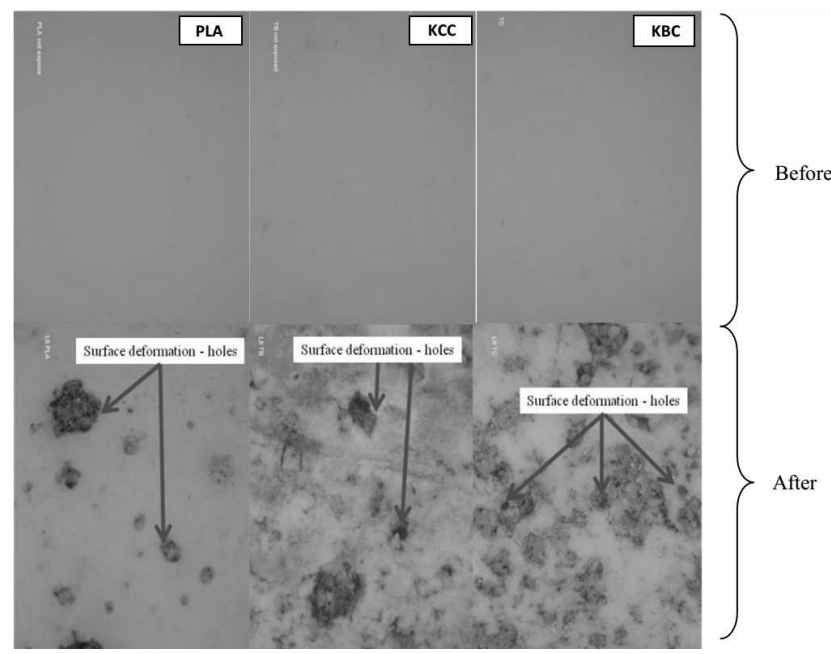

Fig. 5. Microscopic observation of landfill burial samples of PLA, KCC and KBC.

the PLA film was brittle even after 1 month of soil burial. From Fig. 5, the frequency of surface deformation was massively observed on KCC followed by KBC and PLA. Surface deformation on KBC and KCC was expected due to the incorporation of natural fibre, however, concerning the pure PLA, it shows that pure PLA is able to self degrade under soil burial environment, although at a lower rate, as shown in Fig. 4.

\section{Conclusions}

Degradation of PLA/kenaf composites was studied by landfill burial test. KBC and KCC shows higher degradation rate (higher weight loss) than the pure PLA, although a small amount of fibre was incorporated into the PLA composite. It is proven that even presence of small amount of cellulosic material, could lead to degradation of polymer composites. Percentage of weight loss shows a higher weight loss for KBC and KCC (approximately $15.9 \%$ and $17.1 \%$ ) after six months of landfill burial, compared to pure PLA. Additionally, the microscopic observation shows changes on the surface where holes and rougher surface of exposed samples were detected. Results reveal that $\mathrm{KBC}, \mathrm{KCC}$ and pure PLA could be degraded under dry and wet seasons of the Malaysian weather.

\section{References}

[1] J. Lunt, Polym. Degrad. Stab. 59, 145 (1998).

[2] R.E. Drumright, P.R. Gruber, D.E. Henton, $A d v$. Mat. 12, 1841 (2000).

[3] M. Itavaara, S. Karjomaa, J. Selin, Chemosphere 46, 879 (2002).

[4] R.E. Farrel, T.J. Adamczyk, D.C. Broe, J.S. Lee, B.L. Briggs, R.A. Gross, S.P. McCarthy, S. Goodwin, ACS Symp. Series 786, 337 (2001).

[5] X. Zhang, M. Espiritu, A. Bilyk, L. Kurniawan, Polym. Degrad. Stab. 93, 1964 (2008).

[6] R.A. Auras, B. Harte, S. Selke, Macromol. Biosci. 4, 835 (2004).

[7] Y. Tokiva, A. Jarerat, Biotech. Letter 26, 771 (2004).

[8] M. Kunioka, F. Nimomiya, M. Funabashi, Polym. Degrad. Stab. 91(9), 1919 (2006).

[9] G. Kale, R. Auras, S.P. Singh, R. Nayaran, Polym. Test. 26, 1049 (2007).

[10] E. Rudnik, D. Briassoulis, Ind. Crops Prod. 33, 648 (2011).

[11] K. Fukushima, C. Abbate, D. Tabuani, M. Gennari, G. Camino, Polym. Degrad. Stab. 94, 1646 (2009).

[12] S.R. Andersson, M. Hakkarainen, S. Inkinen, A. Sodergard, A.C. Albertsson, Biomacromol. 11, 1067 (2010).

[13] Y.Q. Zhao, H.Y. Cheung, K.T. Lau, C.L. Xu, D.D. Zhao, H.L. Li, Polym. Degrad. Stab. 95, 1978 (2010).

[14] X. Lu, M.Q. Zhang, M.Z. Rong, D.L. Yue, G.C. Yang, Comp. Sci. Tech. 64, 1301 (2004).

[15] L. Liu, S. Li, H. Garreau, M. Vert, Biomacromol. 1, 350 (2000).

[16] S. Li, S. McCarthy, Biomat. 20, 35 (1999).

[17] R.L. Shogren, W.M. Doane, D. Garlotta, J.W. Lawton, J.L. Willett, Polym. Degrad. Stab. 79, 405 (2003). 